Open Access

\title{
The pre-ECMO simplified acute physiology score II as a predictor for mortality in patients with initiation ECMO support at the emergency department for acute circulatory and/or respiratory failure: a retrospective study
}

Kun II Kim ${ }^{1 \dagger}$, Hee Sung Lee ${ }^{1 \dagger}$, Hyoung Soo Kim², Sang Ook Ha ${ }^{3}$, Won Yong Lee ${ }^{2}$, Sang Jun Park ${ }^{2}$, Sun Hee Lee ${ }^{2}$, Tae Hun Lee ${ }^{4}$, Jeong Yeol Seo ${ }^{4}$, Hyun Hee Choi ${ }^{5}$, Kyu Tae Park ${ }^{5}$, Sang Jin Han ${ }^{6}$, Kyung Soon Hong ${ }^{5}$,

Sung Mi Hwang ${ }^{7}$ and Jae Jun Lee ${ }^{7}$

\begin{abstract}
Background: In the emergency department (ED), extracorporeal membrane oxygenation (ECMO) can be used as a rescue treatment modality for patients with refractory circulatory and/or respiratory failure. Serious consideration must be given to the indication, and the PRESERVE and RESP scores for mortality have been investigated. However these scores were validated to predict survival in patients who received mainly veno-venous (W) ECMO in the intensive care unit. The aim of the present study was to investigate the factors that predicted the outcomes for patients who received mixed mode (veno-arterial [VA] and W) ECMO support in the ED.

Methods: This single center retrospective study included 65 patients who received ECMO support at the ED for circulatory or respiratory failure between January 2009 and December 2013. Pre-ECMO SAPS II and other variables were evaluated and compared for predicting mortality.

Results: Fifty-four percent of patients received ECMO-cardiopulmonary resuscitation (E-CPR), $31 \%$ received VA and $\mathrm{V}$-AV ECMO, and $15 \%$ received W ECMO. The 28-day and 60-month mortality rates were $52 \%$ and $63 \%$. In the multivariate analysis, only the pre-ECMO Simplified Acute Physiology Score II (SAPS II) (odd ratio: 1.189, $95 \%$ confidence interval: 1.032-1.370, $p=0.016$ ) could predict the 28-day mortality. The area under the receiver operating characteristic curve and the optimal cutoff value for pre-ECMO SAPS II in predicting 28-day mortality was 0.852 (95\% Cl: $0.753-0.951, p<0.001$ ) and 80 (sensitivity of $97.1 \%$ and specificity of $71.0 \%$ ), respectively. Validation of the 80 cutoff value revealed a statistically significant difference for the 28-day and 60-month mortality rates in the overall, $\mathrm{E}-\mathrm{CPR}$, and VA groups (28-day: $p<0.001, p=0.004, \mathrm{p}=0.005$; 60-month: $p<0.001, p=0.004, p=0.020$ ). In the Kaplan-Meier analysis, the 28-day and 60-month survival rates were lower among the patients with a pre-ECMO SAPS II of $\leq 80$, compared to those with a score of $>80$ (both, $p<0.001$ ).

(Continued on next page)
\end{abstract}

\footnotetext{
* Correspondence: cskhs99@hallym.or.kr

${ }^{\dagger}$ Equal contributors

2Department of Emergency Medicine, Hallym University Medical Center, Kyoungki-do, South Korea

Full list of author information is available at the end of the article
} 
(Continued from previous page)

Conclusion: The pre-ECMO SAPS II could be helpful for identifying patients with refractory acute circulatory and/or respiratory failure who will respond to ECMO support in the ED.

Keywords: SAPS II, Extracorporeal membrane oxygenation, Emergency department, Circulatory failure, Respiratory failure

\section{Background}

Patients with acute circulatory and/or acute respiratory failure in the emergency department (ED) must be diagnosed rapidly and accurately, and an optimal treatment plan must be established according to their initial diagnosis. Unfortunately, many patients' condition rapidly deteriorates before an accurate diagnosis, despite the use of a vasopressor and mechanical ventilator. In this context, extracorporeal membrane oxygenation (ECMO) can be used as a rescue treatment to stabilize their condition and provide additional time to reach an accurate diagnosis, which ultimately leads to improvements in survival rates.

ECMO has recently become increasingly popular for treating patients with acute heart and/or respiratory failure or arrest who do not respond to conventional treatment [1-9]. However, serious consideration must be given to the therapeutic indication for ECMO, due to the high economic cost and labor intensive nature. Therefore, several mortality risk score systems (PRESERVE and RESP) have been investigated for identifying patients who are indicated for ECMO support $[10,11]$. However these scores were validated to predict survival in patients who received mainly veno-venous (VV) ECMO in the intensive care unit (ICU). The aim of the present study was to investigate the factors that predict the outcomes for patients who received mixed mode (veno-arterial [VA] and VV) ECMO support in the ED for circulatory or respiratory failure.

\section{Methods \\ Patient enrollment criteria}

This study was approved by the institutional review board of Hallym University Chuncheon Sacred Heart Hospital, and the requirement for informed consent was waived, due to the retrospective design. Our search identified 65 patients with acute circulatory and/or respiratory failure who did not respond to conventional treatment and received ECMO at the ED between January 2009 and December 2013 (Fig. 1). The indications for VA ECMO were (1) refractory cardiogenic, septic, or neurogenic shock, with a systolic blood pressure of $<80 \mathrm{mmHg}$, despite appropriate conventional treatment; (2) cardiac arrest that did not present with return of spontaneous circulation within 10 min of cardiopulmonary resuscitation (CPR); and (3) recurrent cardiac arrest within 20 min of return of spontaneous circulation after CPR. The indications for VV ECMO were (1) a ratio of $<100$ for partial arterial oxygen pressure to fractional inspired oxygen concentration $\left(\mathrm{PaO}_{2} / \mathrm{FiO}_{2}\right)$ on $\mathrm{FiO}_{2} 1.0$ or (2) a pH of $<7.20$ that was caused by the lack of correction in $\mathrm{CO}_{2}$ retention, despite appropriate conventional treatment for acute respiratory failure.

In addition, patients who were predicted to require a high dose of inotropic agents (norepinephrine $>0.25 \mu \mathrm{g} / \mathrm{kg} / \mathrm{min}$ ) during VV ECMO (due to acute respiratory failure) received V-AV ECMO. Furthermore, if a patient presented with upper limb hypoxia, due to a lack of lung or heart function recovery in the VA mode, they received $\mathrm{V}$-AV or VV ECMO. Patients did not receive ECMO in cases of unwitnessed cardiac arrest, terminal malignancy, or if they

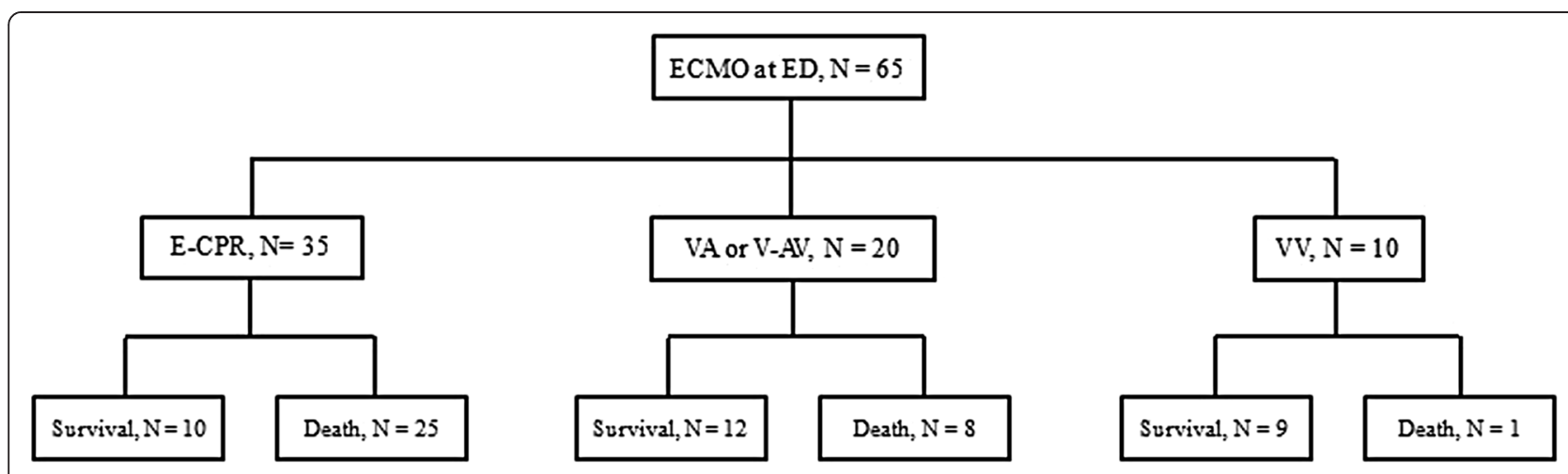

Fig. 1 Flow chart of the study population and outcomes at 28 days. ED: emergency department; ECMO: extracorporeal membrane oxygenation, E-CPR: ECMO-cardiopulmonary resuscitation, W: veno-venous, V-AV: veno-arteriovenous 
were unlikely to regain normal function after general recovery.

\section{Patient data collection}

Electronic medical records were reviewed. Pre-ECMO characteristics (age, sex, body mass index, medical history, diagnosis, arrest [location, CPR time and complication, ECMO-cardiopulmonary resuscitation], laboratory finding, SOFA, pre-ECMO SAPS II, and door-to-ECMO time) and duration and post-ECMO characteristics (ECMO modes, anticoagulation, duration, continuous renal replacement therapy, transfusion, length of stay) were retrospectively calculated.

\section{ECMO equipment}

Three types of centrifugal pumps were used for ECMO. Until May 2010, the Capiox Emergency Bypass System ${ }^{\circ}$ (Terumo, Inc., Tokyo, Japan) and Bio-pump (Medtronic Inc., Minneapolis, USA) were used at our institution. However, since June 2010, the Centrifugal Rotaflow Pump ${ }^{\circ}$ (Maquet Inc., Hirrlingen, Germany) has been used for most patients. The cannulae were $17-21-\mathrm{Fr}$ arterial cannulae $\left(\mathrm{DLP}^{\circ}\right.$, Biomedicus ${ }^{\circ}$; Medtronic Inc., or $\mathrm{RMI}^{\circ}$; Edward's Lifesciences LLC, Irvine, CA, USA) and $17-28-\mathrm{Fr}$ venous cannulae $\left(\right.$ DLP $^{\circ}$, Biomedicus ${ }^{\circ}$, Medtronic Inc., or $\mathrm{RMI}^{\circ}$, Edward's Lifesciences LLC), depending on the patient's size.

\section{ECMO management}

After the injection of a $50-80 \mathrm{IU} / \mathrm{kg}$ heparin bolus, all ECMO patients underwent cannulation using the Seldinger technique in the cardiac catheterization laboratory, which is located next to the ED. When nafamostat mesilate (SK Chemicals Life Science Biz., Seoul, Korea licensed by Torii Pharmaceutical Co., LTD, Tokyo, Japan) was used for anticoagulation during the ECMO, the maintenance dose was held at $0.4-1.5 \mathrm{mg} / \mathrm{kg} / \mathrm{h}$ to maintain a partial thromboplastin time of 60-80 s [12]. Patients with pulmonary embolism or who had not been placed on continuous renal replacement therapy received heparin. The ECMO flow was held at 3.0-4.0 L/min to maintain a mean blood pressure of $>60 \mathrm{mmHg}$, and we administered norepinephrine or dopamine, as necessary, to maintain the appropriate arterial blood pressure. Among the patients who received E-CPR, those who exhibited a Glasgow Coma Scale score of $<9$ (eye response: eyes opening to speech, motor: obeys commands, verbal: intubated state) after arriving at the intensive care unit (ICU) were subjected to hypothermic therapy, without the use of sedative drugs, by maintaining their body temperature at $33-34{ }^{\circ} \mathrm{C}$ for $24 \mathrm{~h}$ [13]. Patients who recovered to a Glasgow Coma Scale of $\geq 9$ were immediately placed on sedatives to increase their body temperature in increments of $0.2{ }^{\circ} \mathrm{C} / \mathrm{h}$. During $\mathrm{ECMO}$, the ventilator mode was maintained at a tidal volume of $5 \mathrm{~mL} / \mathrm{kg}$, the respiratory rate was maintained at $10 / \mathrm{min}$, and the positive end expiratory pressure was maintained at 4-8 $\mathrm{cm} \mathrm{H}_{2} \mathrm{O}$. The $\mathrm{FiO}_{2}$ was held at $0.21-1.0$ to maintain 88-100 \% arterial oxygen saturation. During VVECMO, patients with a clear mental state and a predicted capacity for sputum expectoration were extubated, and oxygen was supplied through a nasal cannula at $3 \mathrm{~L} / \mathrm{min}$ with awakening ECMO. Patients with acute myocardial infarction (AMI) received percutaneous coronary intervention (PCI) before or after the ECMO. On the day of the PCI, the patients received clopidogrel $(300 \mathrm{mg})$ and aspirin $(250 \mathrm{mg})$, and also received clopidogrel $(75 \mathrm{mg})$ and aspirin (100-200 mg) on the following day. Hematocrit levels and platelet counts of $30-35 \%$ and $50,000-80,000 / \mathrm{mL}$, respectively, were targeted, and a blood transfusion was performed if the level(s) fell below the designated threshold. In cases of heart function recovery without lung function recovery during VA-ECMO, which led to upper body hypoxia $\left(\mathrm{PaO}_{2}\right.$ of $\left.<50 \mathrm{mmHg}\right)$, the ECMO mode was changed to V-AV ECMO if the left ventricle ejection fraction (LVEF) was $<30 \%$ (as assessed via two-dimensional echocardiography), or to VV ECMO if the LVEF was $>30 \%$.

The use of VA ECMO was discontinued when echocardiography revealed an LVEF of $>30 \%$ at an ECMO flow of $1 \mathrm{~L} / \mathrm{min}$. The use of VV ECMO was discontinued if the arterial blood gas analysis indicated a $\mathrm{pH}$ of $>7.25$, a $\mathrm{PaO}_{2}$ of $80-120 \mathrm{mmHg}$, and a $\mathrm{PaCO}_{2}$ of $35-45 \mathrm{mmHg}$ at a flow of $1-2 \mathrm{~L} / \mathrm{min}$. To patients who were receiving VV ECMO, we delivered a $\mathrm{FiO}_{2}$ of 0.21 (supplied via the gas blender) and $0 \mathrm{~L} / \mathrm{min}$ sweep gas, using ventilator settings of: VT of $6 \mathrm{~mL} / \mathrm{kg}$, a respiratory rate of $12 / \mathrm{min}$, and $8 \mathrm{~cm}$ of $\mathrm{H}_{2} \mathrm{O}$ positive end expiratory pressure. Alternatively, a $\mathrm{FiO}_{2}$ of 0.6 or oxygen $(3 \mathrm{~L} / \mathrm{min})$ was delivered via a nasal cannula to patients who were awake. Successful ECMO weaning was defined as patient survival of $>24 \mathrm{~h}$ after ECMO removal, and the primary end-point was defined as patient survival of $>28$ days.

\section{Calculation of pre-ECMO SAPS II}

SAPS II is a severity score and mortality estimation tool that was developed for patients in medical or surgical ICUs $[14,15]$. In this tool, data regarding the worst physiological variables are collected within the first $24 \mathrm{~h}$ of ICU admission. In this present study, we modified the SAPS II tool to collect the relevant pre-ECMO data for patients in the ED by collecting data regarding the worst variables within the first $24 \mathrm{~h}$ after admission to the ED. In cases of arrest, the heart rate and Glasgow Coma Scale variables were scored at the lowest value (heart rate of $<40$ beats/min and Glasgow Coma Scale score of $<6$ ). If the patient died within $24 \mathrm{~h}$, the urine output variable was estimated by multiplying the hourly urine output (total urine output divided by the total time) by 24 . 
Table 1 The patient characteristics before extracorporeal membrane oxygenation

\begin{tabular}{|c|c|}
\hline Characteristics & Number \\
\hline Sex, male & 52 \\
\hline Age, years & $56.0(42.5,71.5)$ \\
\hline Body mass index $\left(\mathrm{kg} / \mathrm{m}^{2}\right)$ & $22.9(21.8,26.1)$ \\
\hline \multicolumn{2}{|l|}{ Medical History } \\
\hline Hypertension & 25 \\
\hline Diabetes & 22 \\
\hline Coronary artery disease & 4 \\
\hline Chronic kidney disease & 3 \\
\hline Cerebral vascular accident & 3 \\
\hline Pulmonary disease & 1 \\
\hline \multicolumn{2}{|l|}{ Diagnosis, n (survivors) } \\
\hline Acute cardiac failure & $42(18)$ \\
\hline Acute myocardial infarction & $28(10)$ \\
\hline Pulmonary thromboembolism & $4(3)$ \\
\hline Unknown cardiac arrest & $3(0)$ \\
\hline Other* & $7(5)$ \\
\hline Refractory septic shock & $9(3)$ \\
\hline Neurogenic circulatory failure & $1(0)$ \\
\hline Acute respiratory failure & $13(10)$ \\
\hline Traumatic respiratory failure & $8(8)$ \\
\hline Sepsis related respiratory failure & $3(1)$ \\
\hline Neurogenic pulmonary edema & $1(1)$ \\
\hline Status asthmaticus & $1(0)$ \\
\hline Arrest, n (survivors) & $51(17)$ \\
\hline Out of hospital, n (survivors) & $35(15)$ \\
\hline ED, $n$ (survivors) & $16(2)$ \\
\hline CPR time, min & $55(20,72)$ \\
\hline CPR-related complications $^{\ddagger}$ & $22(34 \%)$ \\
\hline E-CPR, n (survivors) & $35(10)$ \\
\hline \multicolumn{2}{|l|}{ Pre-ECMO laboratory findings } \\
\hline $\mathrm{pH}$ & $7.09(6.94,7.23)$ \\
\hline $\mathrm{PaO} 2 / \mathrm{FiO} 2$ & $54.84(28.9,80.0)$ \\
\hline CK-MB & $4.04(1.80,12.25)$ \\
\hline Troponin-I & $0.1(0.03,0.86)$ \\
\hline BUN & $15.4(11.85,20.85)$ \\
\hline Creatinine & $1.20(0.95,1.40)$ \\
\hline Total bilirubin & $0.87(0.60,1.38)$ \\
\hline AST & $55.0(32.5,120.5)$ \\
\hline ALT & $40.0(24.0,106.5)$ \\
\hline Lactate & $8.75(6.10,12.73)$ \\
\hline IABP & 10 \\
\hline SOFA score & $13(11,14)$ \\
\hline Pre-ECMO SAPS ॥ & $88(70,97)$ \\
\hline
\end{tabular}

Table 1 The patient characteristics before extracorporeal membrane oxygenation (Continued)

\begin{tabular}{|c|c|}
\hline Door to ECMO time ${ }^{\dagger}, \min$ & $93.0(56.5,182.0)$ \\
\hline $\mathrm{E}-\mathrm{CPR}$ & $62.0(49.0,95.0)$ \\
\hline Veno-arterial & $157.0(84.0,348.0)$ \\
\hline Veno-venous & $126.0(102.0,206.0)$ \\
\hline \multicolumn{2}{|c|}{$\begin{array}{l}\text { Continuous variables are reported as median (interquartile range) } \\
C P R \text { cardiopulmonary resuscitation, ED emergency department, E-CPR } \\
\text { extracorporeal cardiopulmonary resuscitation, ECMO extracorporeal membrane } \\
\text { oxygenation, BUN blood urea nitrogen, AST aspartate transaminase, } A L T \\
\text { alanine transaminase, IABP intraaortic balloon pump, SOFA Sequential Organ } \\
\text { Failure Assessment, SAPS II Simplified Acute Physiology Score II } \\
{ }^{*} \text { Hypothermia, malignant arrhythmia, dilated cardiomyopathy, ischemic } \\
\text { cardiomyopathy, commotio cordis, abdominal aortic aneurysm rupture } \\
{ }^{\dagger} \text { Door to ECMO time: the time from arrival at the ED to ECMO implantation } \\
{ }^{\ddagger} \text { Hypoxic brain damage, hemothorax, pulmonary hemorrhage, chest wall } \\
\text { compartment syndrome, chylothorax }\end{array}$} \\
\hline
\end{tabular}

\section{Statistical analysis}

Statistical analyses were performed using IBM SPSS software (version 21; IBM Corp., Armonk, NY, USA), and differences with a $\mathrm{p}$-value of $<0.05$ were considered statistically significant. The Mann-Whitney $U$ test was used to evaluate continuous variables, and Pearson's chi square test or Fisher's exact test was used for categorical variables. To identify the independent factors that were associated with patient death, we used univariate and multivariate stepwise logistic regression analysis models. The pre-ECMO SAPS II, which was the only significant factor in the multivariate stepwise logistic regression analyses, was subjected to receiver operating characteristic curve (ROC) analysis to identify the optimum cutoff value. Using this cutoff score, we validated the performance of the pre-ECMO SAPS II for predicting 28-day and 60-month mortality, according to the indication, and confirmed the 28-day and 60-month cumulative survival rates using Kaplan-Meier analysis.

\section{Results}

Baseline and clinical characteristics of the study patients

Among the 65 patients who were included in this presented study, 35 (53.8\%) patients underwent E-CPR, 20 (30.8\%) underwent VA or V-AV ECMO, and 10 (15.4\%) underwent VV ECMO. The 28-day survival rates were $28.6 \%$ in the E-CPR group, $60 \%$ in the VA or V-AV ECMO group, and $90 \%$ in the VV ECMO group (Fig. 1).

The patients' pre- and during-ECMO characteristics are listed in Tables 1 and 2. The median patient age was 56 years (52 men, 13 women). Regarding the indications for ECMO, AMI (28 patients, $43.1 \%$ ) was the most common cause, and was followed by refractory septic shock and traumatic respiratory failure. Among the 51 patients with arrest, the median duration of CPR was $55 \mathrm{~min}$ and E-CPR was performed in 35 patients. The overall survival rate in the VV mode was higher than 
Table 2 The characteristics of survivors and non-survivors during extracorporeal membrane oxygenation support

\begin{tabular}{|c|c|c|c|}
\hline & Non-survivors & Survivors & $p$-value \\
\hline & $n=34$ & $n=31$ & \\
\hline ECMO modes & & & 0.018 \\
\hline VA mode & 31 & 21 & \\
\hline Change from VA to W & - & 1 & \\
\hline Change from VA to V-AV & 1 & - & \\
\hline $\begin{array}{l}\text { Change from VA to V-AV, and } \\
\text { then to } \mathrm{W}\end{array}$ & - & 1 & \\
\hline V-AV mode & 2 & 1 & \\
\hline Change from $\mathrm{V}-\mathrm{AV}$ to $\mathrm{W}$ & 1 & - & \\
\hline$W$ mode & 1 & 9 & \\
\hline Anticoagulants & & & 0.179 \\
\hline Nafamostat mesilate & 30 & 26 & \\
\hline Heparin & 2 & 5 & \\
\hline ECMO run of $\leq 24 \mathrm{~h}$ & 9 & 1 & $0.014^{a}$ \\
\hline Surgery after ECMO & - & 4 & $0.046^{\mathrm{a}}$ \\
\hline ECMO duration, $\mathrm{h}$ & $72.5(23.8,118)$ & $143.0(93,212)$ & 0.001 \\
\hline CRRT & $18(60 \%)$ & $5(71 \%)$ & $0.687^{a}$ \\
\hline Daily blood transfusion, unit/d & - & - & \\
\hline Packed red blood cells & $1.9(0.9,3.0)$ & $1.0(0.7,1.3)$ & 0.002 \\
\hline Fresh frozen plasma & $1.0(0.5,2.4)$ & $0.3(0.0,0.7)$ & 0.001 \\
\hline Platelet concentrate & $1.7(0.0,5.0)$ & $1.3(0.0,3.3)$ & 0.823 \\
\hline \multicolumn{4}{|l|}{ Length of stay } \\
\hline ICU & $4(2,7)$ & $18(11,30)$ & $<0.001$ \\
\hline Hospital & $4(2,7)$ & $28(20,51)$ & $<0.001$ \\
\hline
\end{tabular}

${ }^{\mathrm{a}}$ Fisher's exact test. Continuous variables are reported as median (interquartile range)

$E C M O$ extracorporeal membrane oxygenation, $V A$ veno-arterial, $W V$ veno-venous, $V-A V$ veno-arteriovenous, $C R R T$ continuous renal replacement therapy, ICU intensive care unit

Surgeries included two pulmonary artery thromboembolectomies, a left upper lobectomy, and an aneurysm clipping via a left frontotemporal craniectomy

that in the VA or V-AV mode (21/52, $40 \%$ vs. 10/13, $77 \%, p=0.018)$. Compared to the non-survivor group, the survivor group had a longer ECMO duration (72.5 $\mathrm{min}$ vs. $143.0 \mathrm{~min}, p=0.001$ ) and fewer blood transfusions (packed red blood cells: 1.9 units/day vs. 1.0 units/day, $p=0.002$; fresh frozen plasma: 1.0 units/day vs. 0.3 units/day, $p=0.001$ ). However, there were no differences in the use of CCRT, type of anticoagulation treatment, and complications between the survivor and non-survivor groups (Table 3).

\section{Predictors for mortality in patients with ECMO support at the ED}

In the logistic regression analyses, all 28-day variables that were under consideration (hypertension, cardiac arrest on arrival, E-CPR, CPR time, ECMO VA mode, lactate, SOFA score, pre-ECMO SAPS II, and CPR-related
Table 3 A comparison of complications among survivors and non-survivors

\begin{tabular}{llll}
\hline & Non-survivors & Survivors & $\mathrm{p}$-value \\
& $\mathrm{n}=34$ & $\mathrm{n}=31$ & \\
\hline Cannula-related complications & 2 & 2 & $1.000^{\mathrm{a}}$ \\
Cannula site bleeding & 1 & - & \\
Leg ischemia & 1 & 1 & \\
Thrombosis & - & 1 & \\
Acute renal failure & 12 & 9 & 0.590 \\
SIRS & 1 & - & $1.000^{\mathrm{a}}$ \\
Gastrointestinal bleeding & 3 & 2 & $1.000^{\mathrm{a}}$ \\
Cholecystitis & - & 1 & $0.477^{\mathrm{a}}$ \\
Intracranial hemorrhage & 2 & - & $1.000^{\mathrm{a}}$ \\
\hline aFisher's exact test. SIRS, systemic inflammatory response syndrome
\end{tabular}

complications) were significantly higher in the nonsurvivors, compared to the values in the survivors. However, in the multivariate analysis, only pre-ECMO SAPS II was a statistically significant predictor (odds ratio $[\mathrm{OR}]: 1.189 ; 95 \%$ confidence interval [CI]: 1.032-1.370; $p=0.016$ ) (Table 4).

The clinical relevance of pre-ECMO SAPS II as a predictor for 28-day mortality was further confirmed by the subsequent ROC analyses. The area under the ROC curve for pre-ECMO SAPS II was 0.852 (95\% CI: $0.753-0.951, p<0.001)$, and the optimal cutoff value for pre-ECMO SAPS II was 80 (sensitivity of $97.1 \%$ and specificity of $71.0 \%$ ), respectively (Fig. 2).

\section{Validating the performance of pre-ECMO SAPS II for 28-day and 60-month mortality}

The cutoff value of 80 exhibited a statistically significant difference for the 28-day and 60-month mortality rates in the overall, E-CPR, and VA groups (28-day: $p<0.001, p=$ $0.004, p=0.005 ;$ 60-month: $p<0.001, p=0.004, p=$ 0.020) (Table 5). In the Kaplan-Meier analysis, the 28day and 60-month cumulative survival rates were lower among the patients with a pre-ECMO SAPS II of $\leq 80$, compared to those with a pre-ECMO SAPS II of $>80$ (both, $p<0.001$ ) (Fig. 3).

\section{Discussion}

The present study's results demonstrated that preECMO SAPS II is an independent predictor for 28-day mortality among patients with ECMO support at the ED. In the validation of its performance, the cutoff value of 80 exhibited statistically significant differences for the 28-day and 60-month mortality rates, especially in the E-CPR and VA ECMO groups. Therefore, the preECMO SAPS II could be helpful for selecting patients with refractory acute circulatory and/or respiratory failure who will respond to ECMO support at the ED. 
Table 4 Univariate and multiple logistic regression analysis of pre-extracorporeal membrane oxygenation predictors of 28-day mortality

\begin{tabular}{|c|c|c|c|c|c|c|}
\hline \multirow[t]{2}{*}{ Variable } & \multicolumn{3}{|c|}{ Univariate analysis } & \multicolumn{3}{|c|}{ Multivariate analysis } \\
\hline & OR & $95 \% \mathrm{Cl}$ & $p$-value & OR & $95 \% \mathrm{Cl}$ & $p$-value \\
\hline Hypertension & 3.857 & $1.312-11.337$ & 0.014 & 1.921 & $0.123-29.942$ & 0.641 \\
\hline Cardiac arrest on arrival & 3.392 & $1.220-9.431$ & 0.019 & 0.012 & $0.000-1.896$ & 0.087 \\
\hline $\mathrm{E}-\mathrm{CPR}$ & 5.833 & $1.998-17.028$ & 0.001 & 1.078 & $0.055-21.099$ & 0.960 \\
\hline CPR time & 1.021 & $1.000-1.041$ & 0.047 & 1.002 & $0.955-1.051$ & 0.941 \\
\hline VAM ECMO & 14.211 & $1.665-121.316$ & 0.015 & 4.626 & $0.023-925.946$ & 0.571 \\
\hline Lactate & 1.334 & $1.109-1.605$ & 0.002 & 1.345 & $0.885-2.045$ & 0.165 \\
\hline SOFA score & 1.430 & $1.146-1.785$ & 0.002 & 1.191 & $0.539-2.633$ & 0.665 \\
\hline CPR related $\mathrm{Cx}$ & 7.594 & $2.181-26.437$ & 0.001 & 1.138 & $0.082-15.840$ & 0.923 \\
\hline Pre-ECMO SAPS ॥ & 1.131 & $1.059-1.207$ & $<0.001$ & 1.189 & $1.032-1.370$ & 0.016 \\
\hline
\end{tabular}

In order to solve the multicollinearity problem, we excluded the variables that are included in SAPS II from the logistic regression analysi $E$ - $C P R$ extracorporeal cardiopulmonary resuscitation, $C P R$ cardiopulmonary resuscitation, $V A$ veno-arterial, $W V$ veno-venous, $E C M O$ extracorporeal membrane oxygenation, SOFA Sequential Organ Failure Assessment, SAPS II Simplified Acute Physiology Score II, Cx complications

The selection of appropriate candidates for ECMO support requires serious consideration, due to the high economic cost and labor intensive nature. A small number of recent studies have attempted to develop a scoring system for identifying these patients. Schmidt et al. evaluated the PRESERVE and RESP scores in their survival prediction model for patients who received ECMO support in the ICU $[10,11]$. However, there are several limitations to applying these systems for patients in the

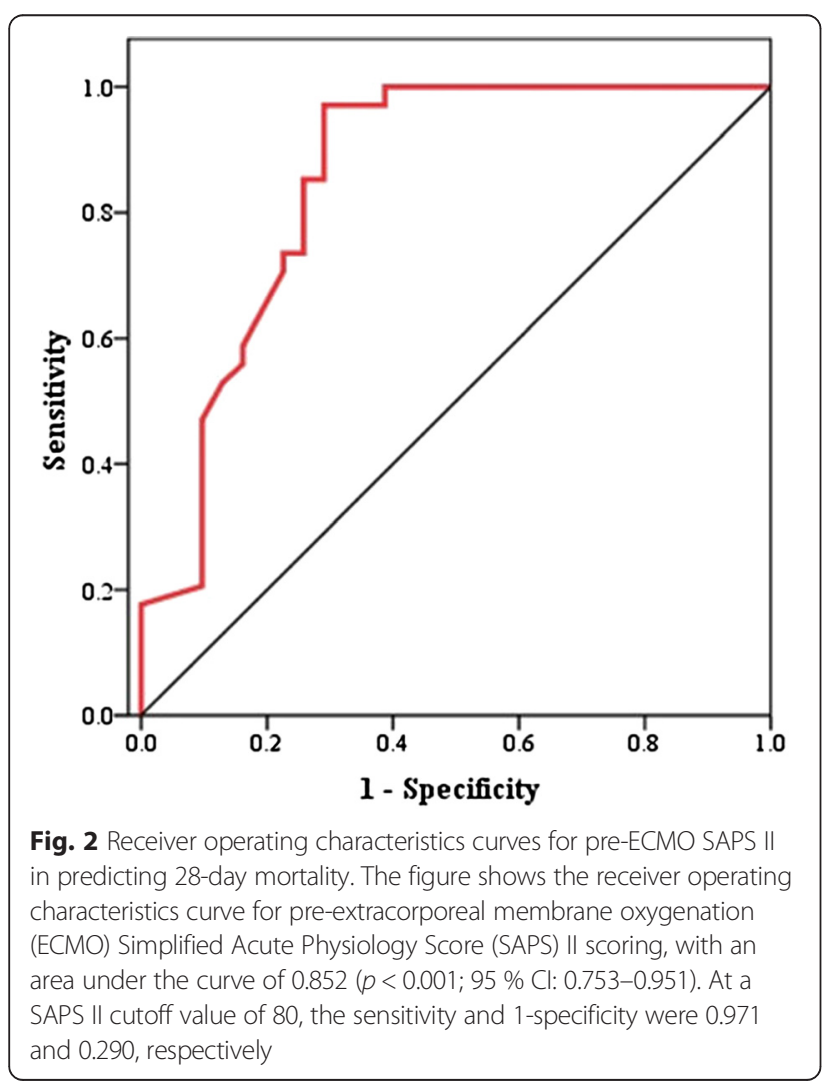

ED. First, those studies only enrolled patients with respiratory failure, which inevitably led to most of the ECMO being VV mode (PRESERVE VA mode: 133/ 140 [95\%] and RESP VA mode: 1,928/2,355 [82 \%]). However, similar to our results, VA modes were applied more than VV modes for patients who received ECMO support in the ED. Second, during E-CPR and VA ECMO, it is difficult to assess mechanical ventilation duration and settings. Third, Klinzing et al. demonstrated that the RESERVE and RESP scoring systems failed to predict mortality for patients who were receiving VA ECMO [16]. In the present study, preECMO SAPS II significantly predicted the survival of patients who received VA ECMO and E-CPR. The VV ECMO group exhibited a similar tendency, although the result was not statistically significant.

Our survival rate results demonstrated better outcomes, compared to those of previous studies. We suggest that the discrepancies between these findings may be related to patient enrollment. The majority of indications for VA or V-AV ECMO were for acute cardiac failure, especially AMI, and only a relatively small number of patients with septic shock (which has a poor prognosis) were included. In contrast, the major indication for VV ECMO was traumatic acute respiratory distress syndrome, which has a favorable prognosis.

Given the ongoing progress in the development of ECMO equipment, cannula can easily and safely be implanted into patients' peripheral vessels, which facilitates the rapid application of ECMO. This rapid emergent cardiopulmonary assistance technique can also facilitate the appropriate testing and diagnosis, and thereby allow for appropriate treatment [17-20]. In the present study, $43 \%$ of the patients who received ECMO support at the ED had presented with complications of refractory cardiogenic shock or cardiac arrest due to AMI. These 
Table 5 Validation of SAPS II for 28-day and 60-month mortality according to the indication

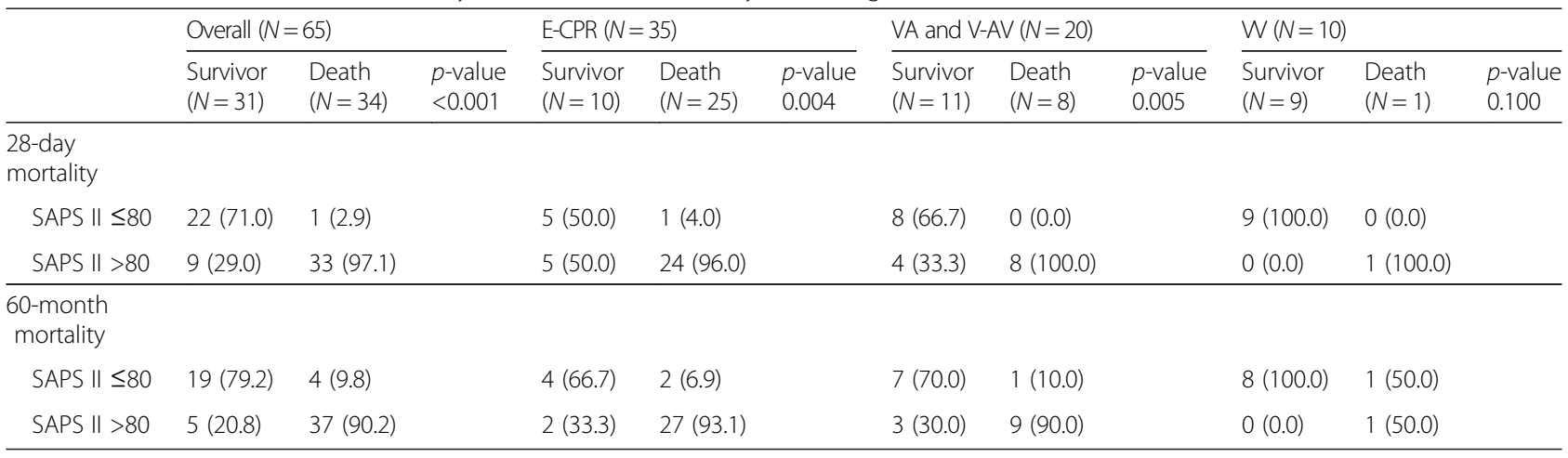

patients underwent PCI with ECMO, which provided a $35.7 \%$ patient survival rate. Therefore, AMI that is accompanied by cardiogenic shock, or cases of cardiac arrest that present with a high risk of mortality when using conventional treatment methods, may be suitable for ECMO, which can facilitate appropriate diagnosis and treatment, and improve patient survival rates $[6,21-25]$. Furthermore, other studies have reported that, in cases of in- and out-of-hospital cardiac arrest with cardiac origin, E-CPR provided a survival rate of $32.1-34.1 \%$, which is higher than that obtained using conventional CPR $[5,7,26]$.

Among the four patients with acute massive pulmonary embolism who received ECMO in our study, one patient presented with out-of-hospital cardiac arrest and was diagnosed with a massive pulmonary embolism after the E-CPR. Unfortunately, this patient subsequently died of hypoxic brain damage. Two of the three survivors underwent pulmonary embolectomy after the ECMO, while the other surviving patient underwent successful medical treatment. For patients in near-fatal condition (due to massive pulmonary embolisms), the survival rate of medical or surgical treatment after ECMO is $62-70 \%$ $[27,28]$. In these cases, the clinical decision to administer ECMO, the reversibility of the underlying disease, and the patient's condition after ECMO all affect the prognosis, and an aggressive diagnosis and treatment of the underlying disease are critical to patient survival [22].

In this present study, $33 \%$ of the patients with acute sepsis-related circulatory and/or respiratory failure
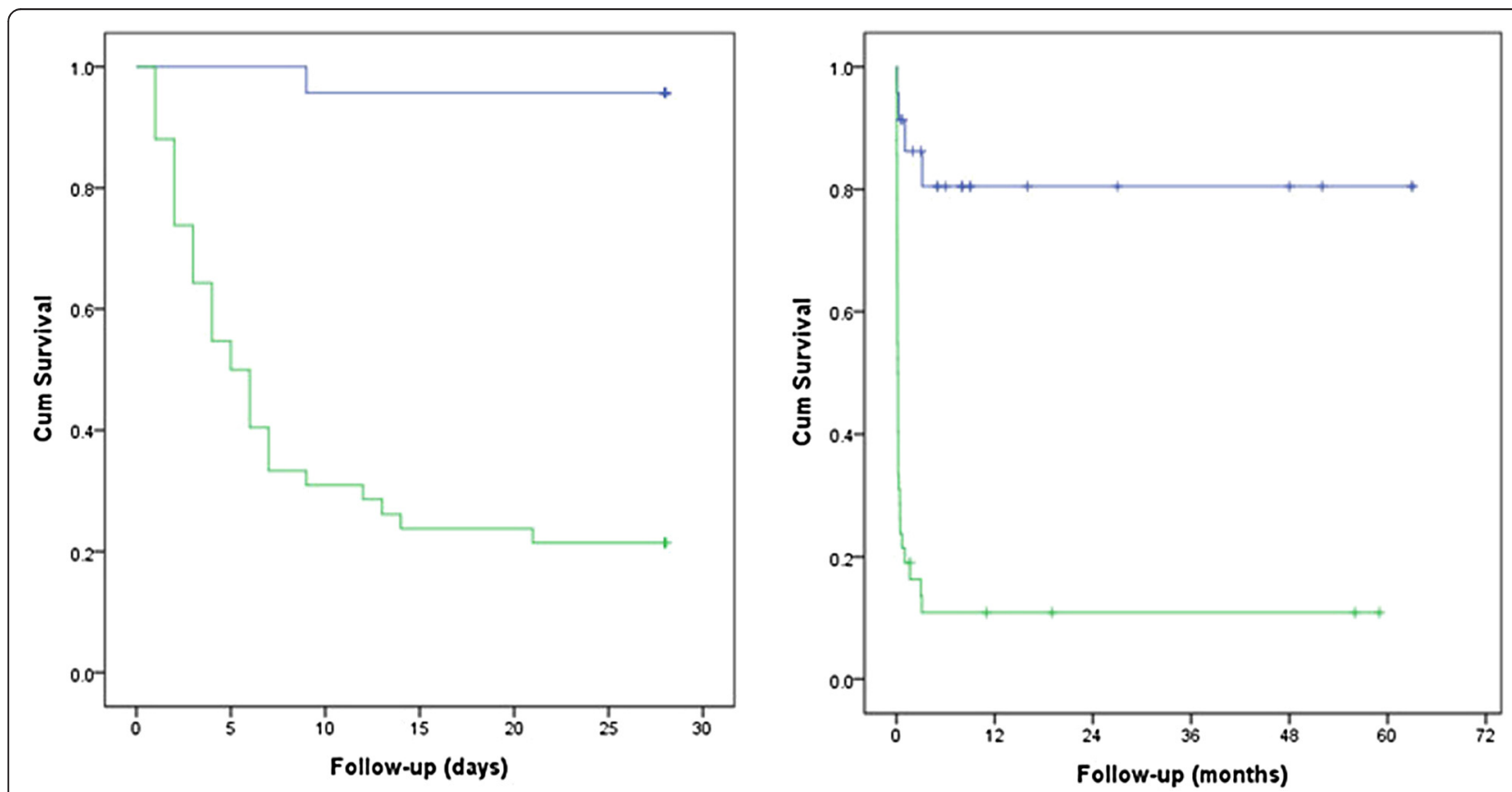

Fig. 3 Kaplan-Meier analysis for a pre-ECMO SAPS II score of 80 in predicting 28-day and 60-month mortality. The risk of 28-day and 60-month mortality among patients with SAPS II of $\leq 80$ was lower than that of patients with SAPS $\|$ of $>80$ (both, $p<0.001$ ) 
ultimately survived. Although the use of ECMO in patients with sepsis remains controversial, it may be effective for use in a select group of patients [8, 29]. In addition, the survival rate for patients with acute respiratory failure was $77 \%(10 / 13)$, compared to $40 \%(21 / 52)$ for patients with acute circulatory failure, which is due to the fact that all 8 patients with traumatic respiratory failure who received ECMO ultimately survived. Therefore, VV ECMO is very effective in treating patients with severe thoracic trauma and acute lung failure from various causes $[3,9]$.

As patients in the ED require rapid diagnosis and treatment, it is difficult to determine appropriate ECMO implantation standards and identify patients with acute cardiopulmonary failure who will not respond to conventional treatment. Although pre-ECMO SAPS II is widely used to predict mortality among ICU patients, this can be also useful for predicting ED patient mortality in cases of cardiovascular and respiratory illnesses [30]. Although the median pre-ECMO SAPS II in our study was 88 , which predicted an extremely high mortality rate $(96.1 \%)$, the 28 -day and 60 -month overall mortality rates for our patients were $52.3 \%$ and $63.1 \%$. Furthermore, these mortality rates for patients with a pre-ECMO SAPS II of $\leq 80$ were $2.9 \%$ and $20.8 \%$, respectively. Therefore, we believe that the pre-ECMO SAPS II score can lead to the appropriate selection of patients for ECMO support, and improve patient outcomes.

This study had several limitations. First, it was based at a single center, which limits the generalizability of the findings. Second, the study population was small, and the underlying diseases were diverse. Third, in VA ECMO, a relatively small number of patients with refractory septic shock were enrolled in this study. Thus, further studies are needed to determine whether our findings can be accurately applied to these patients. Finally, we performed a retrospective analysis, and additional prospective multicenter studies are needed to validate our findings.

\section{Conclusions}

Pre-ECMO SAPS II could be helpful for selecting patients with refractory acute circulatory and/or respiratory failure who will respond to ECMO support at the ED.

\section{Competing interests}

The authors declare that they have no competing interests.

\section{Author contributions}

KIK, HSL, HSO, THL, JYS and HSK have contributed to the conception and design of the study, acquisition and analysis of data. KIK and HSL have drafted the manuscript. HHC, SJH, KSH and KTP helped to draft the manuscript. SHL, SJP, SMH and JJL participated in the design of the study, acquisition of data and performed the statistical analysis. All the authors read and approved the final manuscript.

\section{Author details}

'Department of Thoracic and Cardiovascular Surgery, Hallym University Sacred Heart Hospital, Hallym University Medical Center, 22, Gwanpyeong-ro 170 beon-gil, Donan-gu, Anyang-si, Gyeonggi-do 431-070, South Korea. ${ }^{2}$ Department of Emergency Medicine, Hallym University Medical Center, Kyoungki-do, South Korea. ${ }^{3}$ Department of Emergency Medicine, Hallym University, Chuncheon, South Korea. ${ }^{4}$ Division of Cardiology, Department of Internal Medicine, Hallym University, Chuncheon, South Korea. ${ }^{5}$ Department of Anesthesiology, School of Medicine, Hallym University, Chuncheon, South Korea. ${ }^{6}$ Department of Anesthesiology, School of Medicine, Hallym

University, Chuncheon, South Korea. ${ }^{7}$ Department of Anesthesiology, School of Medicine, Hallym University, Chuncheon, South Korea.

Received: 10 March 2015 Accepted: 14 July 2015

Published online: 17 August 2015

\section{References}

1. Wu MY, Liu YC, Tseng YH, Chang YS, Lin PJ, Wu TI. Pulmonary embolectomy in high-risk acute pulmonary embolism: the effectiveness of a comprehensive therapeutic algorithm including extracorporeal life support. Resuscitation. 2013;84:1365-70.

2. Sakamoto S, Taniguchi N, Nakajima S, Takahashi A. Extracorporeal life support for cardiogenic shock or cardiac arrest due to acute coronary syndrome. Ann Thorac Surg. 2012;94:1-7.

3. Ried M, Bein T, Philipp A, Müller T, Graf B, Schmid C, et al. Extracorporeal lung support in trauma patients with severe chest injury and acute lung failure: a 10-year institutional experience. Crit Care. 2013;17:R110.

4. Pe Peek GJ, Mugford M, Tiruvoipati R, Wilson A, Allen E, Thalanany MM, et al. Efficacy and economic assessment of conventional ventilatory support versus extracorporeal membrane oxygenation for severe adult respiratory failure (CESAR): a multicentre randomised controlled trial. Lancet. 2009:374:1351-63.

5. Maekawa K, Tanno K, Hase M, Mori K, Asai Y. Extracorporeal cardiopulmonary resuscitation for patients with out-of-hospital cardiac arrest of cardiac origin: a propensity-matched study and predictor analysis. Crit Care Med. 2013:41:1186-96.

6. Kagawa E, Dote K, Kato M, Sasaki S, Nakano Y, Kajikawa M, et al. Should we emergently revascularize occluded coronaries for cardiac arrest?: rapid-response extracorporeal membrane oxygenation and intra-arrest percutaneous coronary intervention. Circulation. 2012;126:1605-13.

7. Chen YS, Lin JW, Yu HY, Ko WJ, Jerng JS, Chang WT, et al. Cardiopulmonary resuscitation with assisted extracorporeal life-support versus conventional cardiopulmonary resuscitation in adults with in-hospital cardiac arrest: an observational study and propensity analysis. Lancet. 2008;372:554-61.

8. Bréchot N, Luyt CE, Schmidt M, Leprince $P$, Trouillet JL, Léger $P$, et al. Venoarterial extracorporeal membrane oxygenation support for refractory cardiovascular dysfunction during severe bacterial septic shock. Crit Care Med. 2013;41:1616-26.

9. Biderman $P$, Einav $S$, Fainblut $M$, Stein $M$, Singer $P$, Medalion $B$. Extracorporeal life support in patients with multiple injuries and severe respiratory failure: a single-center experience? J Trauma Acute Care Surg 2013;75:907-12.

10. Schmidt M, Bailey M, Sheldrake J, Hodgson C, Aubron C, Rycus PT, et al. Predicting survival after extracorporeal membrane oxygenation for severe acute respiratory failure. The Respiratory Extracorporeal Membrane Oxygenation Survival Prediction (RESP) score. Am J Respir Crit Care Med. 2014;189:1374-82.

11. Schmidt M, Zogheib E, Rozé H, Repesse X, Lebreton G, Luyt CE, et al. The PRESERVE mortality risk score and analysis of long-term outcomes after extracorporeal membrane oxygenation for severe acute respiratory distress syndrome. Intensive Care Med. 2013;39:1704-13.

12. Han SJ, Kim HS, Kim Kl, Whang SM, Hong KS, Lee WK, et al. Use of nafamostat mesilate as an anticoagulant during extracorporeal membrane oxygenation. J Korean Med Sci. 2011;26:945-50.

13. Rutledge R, Lentz CW, Fakhry S, Hunt J. Appropriate use of the Glasgow Coma Scale in intubated patients: a linear regression prediction of the Glasgow verbal score from the Glasgow eye and motor scores. J Trauma. 1996;41:514-22. 
14. Le Gall JR, Lemeshow S, Saulnier F. A new Simplified Acute Physiology Score (SAPS II) based on a European/North American multicenter study. JAMA. 2013;270:2957-63.

15. Grigore L, Poiata A. Evaluation of sepsis prognosis using Saps II. Revista medico-chirurgicala a Societatii de Medici si Naturalisti din lasi. 2000;104:97-102.

16. Klinzing $S$, Wenger $U$, Steiger $P$, Starck $C T$, Wilhelm M, Schuepbach RA, et al. External validation of scores proposed for estimation of survival probability of patients with severe adult respiratory distress syndrome undergoing extracorporeal membrane oxygenation therapy: a retrospective study. Crit Care. 2015;19:142

17. Kurusz M, Zwischenberger JB. Percutaneous cardiopulmonary bypass for cardiac emergencies. Perfusion. 2002;17:269-77.

18. Nichol G, Karmy-Jones R, Salerno C, Cantore L, Becker L. Systematic review of percutaneous cardiopulmonary bypass for cardiac arrest or cardiogenic shock states. Resuscitation. 2006;70:381-94.

19. Roussel A, Al-Attar N, Alkhoder S, Radu C, Raffoul R, Alshammari M, et al. Outcomes of percutaneous femoral cannulation for venoarterial extracorporeal membrane oxygenation support. Eur Heart J Acute Cardiovasc Care. 2012;1:111-4.

20. Sung K, Lee YT, Park PW, Park KH, Jun TG, Yang JH, et al. Improved survival after cardiac arrest using emergent autopriming percutaneous cardiopulmonary support. Ann Thorac Surg. 2006;82:651-6.

21. Aiba T, Nonogi H, Itoh T, Morii I, Daikoku S, Goto Y, et al. Appropriate indications for the use of a percutaneous cardiopulmonary support system in cases with cardiogenic shock complicating acute myocardial infarction. Jpn Circ J. 2001;65:145-9.

22. Aoyama N, Imai H, Kono K, Kato S, Fukuda N, Kurosawa T, et al. Patient selection and therapeutic strategy for emergency percutaneous cardiopulmonary system in cardiopulmonary arrest patients. Circ J. 2009:73:1416-22.

23. Chen JS, Ko WJ, Yu HY, Lai LP, Huang SC, Chi NH, et al. Analysis of the outcome for patients experiencing myocardial infarction and cardiopulmonary resuscitation refractory to conventional therapies necessitating extracorporeal life support rescue. Critical Care Med. 2006;34:950-7.

24. Combes $A$, Leprince $P$, Luyt CE, Bonnet $N$, Trouillet $J$, Léger $P$, et al. Outcomes and long-term quality-of-life of patients supported by extractorporeal membrane oxygenation for refractory cardiogenic shock. Critical Care Med. 2008;36:1404-11.

25. Wu MY, Tseng YH, Chang YS, Tsai FC, Lin PJ. Using extracorporeal membrane oxygenation to rescue acute myocardial infarction with cardiopulmonary collapse: the impact of early coronary revascularization. Resuscitation. 2013:84:940-5.

26. Shin TG, Choi JH, Jo IJ, Sim MS, Song HG, Jeong YK, et al. Extracorporeal cardiopulmonary resuscitation in patients with inhospital cardiac arrest: A comparison with conventional cardiopulmonary resuscitation. Critical Care Med. 2011;39:1-7.

27. Maggio P, Hemmila M, Haft J, Bartlett R. Extracorporeal life support for massive pulmonary embolism. J Trauma. 2007:62:570-6.

28. Munakata R, Yamamoto T, Hosokawa Y, Tokita Y, Akutsu K, Sato N, et al. Massive pulmonary embolism requiring extracorporeal life support treated with catheter-based interventions. Int Heart J. 2012;53:370-4.

29. Huang CT, Tsai YJ, Tsai PR, Ko WJ. Extracorporeal membrane oxygenation resuscitation in adult patients with refractory septic shock. J Thorac Cardiovasc Surg. 2013;146:1041-6

30. Cosentini R, Folli C, Cazzaniga M, Aliberti S, Piffer F, Grazioli L, et al. Usefulness of simplified acute physiology score II in predicting mortality in patients admitted to an emergency medicine ward. Intern Emerg Med. 2009:4:241-7.

\section{Submit your next manuscript to BioMed Central and take full advantage of:}

- Convenient online submission

- Thorough peer review

- No space constraints or color figure charges

- Immediate publication on acceptance

- Inclusion in PubMed, CAS, Scopus and Google Scholar

- Research which is freely available for redistribution 